\title{
Sleep During Infancy, Inhibitory Control and Working Memory in Toddlers: Findings from the FinnBrain Cohort Study
}

Isabel Morales Muñoz ( $\boldsymbol{Q}$ isabel.morales@thl.fi )

National Institute for Health and Welfare: Terveyden ja hyvinvoinnin laitos https://orcid.org/0000-0002-4718-6768

\section{Saara Nolvi}

University of Turku: Turun Yliopisto

\section{Tiina Mäkelä}

Tampere University: Tampereen Yliopisto

\section{Eeva Eskola}

Turun Yliopisto

Riikka Korja

Turun Yliopisto

Michelle Fernandes

University of Oxford

Hasse Karlsson

Turun Yliopisto

\section{E Juulia Paavonen}

Terveyden ja hyvinvoinnin laitos

\section{Linnea Karlsson}

Turun Yliopisto

\section{Research}

Keywords: sleep, inhibitory control, working memory, infancy, toddlers

Posted Date: December 28th, 2020

DOI: https://doi.org/10.21203/rs.3.rs-131388/v1

License: (c) (i) This work is licensed under a Creative Commons Attribution 4.0 International License. Read Full License

Version of Record: A version of this preprint was published at Sleep Science and Practice on August 15th, 2021. See the published version at https://doi.org/10.1186/s41606-021-00064-4. 


\section{Abstract}

Background: Sleep difficulties are associated with executive functioning (EF) impairment in school-aged children. However, much less is known about how sleep in infancy relates to EF in infants and/or toddlers. The aim of this study was to investigate whether parent-reported sleep patterns in infants at 6 and 12 months of age were associated with inhibitory control (IC) and working memory (WM) performances at 30 months.

Methods: The children were divided into three sleep groups (i.e., "bad sleepers", "intermediate sleepers" and "good sleepers") based on percentile cut-off points in order to have a comprehensive understanding of the direction and nature of the associations between sleep and aspects of EF in early childhood. Sleep was assessed using the Brief Infant Sleep Questionnaire, IC was measured using a modified version of the Snack Delay task ( $\mathrm{N}=425)$, and WM by using the Spin the Pots task $(\mathrm{N}=430)$.

Results: Our results reported an inverted U-shaped association between proportion of daytime sleep at 12 months and IC at 30 months, indicating that average proportions of daytime sleep were longitudinally associated with better IC performance. Furthermore, a linear relation between time awake during night at 12 months and WM at 30 months was found, with more time awake at night associating with worse WM.

Conclusions: Our findings support the hypothesis that sleep disruption in early childhood is associated with the development of later EF and suggest that different sleep difficulties at 12 months distinctively affect WM and IC in toddlers, possibly also in a non-linear manner.

\section{Introduction}

The first years of life are characterized by rapid brain growth and development (Choe et al., 2013; Knickmeyer et al., 2008). Furthermore, these processes are considered to be connected to the development of sleep, which is one of the primary activities of the brain in young children (Dahl, 1996). The most rapid development in sleep organization takes place during the first six months of life, followed by more moderate changes later on (de Weerd \& van den Bossche, 2003; Henderson, France, \& Blampied, 2011).

Adequate sleep is essential for the maintenance of optimal cognitive and emotional functioning, especially in childhood (Astill et al., 2012; Mindell et al., 2011). Of these, executive functions (EF) are particularly sensitive to the effects of childhood sleep problems (Turnbull et al., 2013), with neuroimaging evidence in young adults suggesting that sleep loss affects the frontal lobes more than other brain areas (Cajochen et al., 2001; Finelli et al., 2000; Thomas et al., 2000). Sleep difficulties, such as insufficient sleep or frequent night awakening are associated with worse performance in EF tasks in school-aged children (Astill et al., 2012; Sadeh et al., 2002). More specifically, objective sleep measures such as actigraph-based lower sleep efficiency and longer sleep latency are associated with worse performance in working memory (WM) tasks at all load levels in school-aged children, while actigraph-based shorter sleep duration associates with lower performance in WM tasks at the highest load level only (Steenari et al., 2003). Similar associations have been reported between parental report of increasing sleep problem severity and lower verbal WM scores in school-aged children (Cho et al., 2015). Furthermore, similar findings have been also described in earlier stages of childhood. For instance, maternal-reported insufficient sleep in early school age years (ages 5-7 years) and preschoolers (ages 34 years) has been related to poorer mother- and teacher-report of a range of neurobehavioral processes in middle childhood, including a general measure of EF (Taveras et al., 2017). Finally, in preschoolers, actigraph-based short sleep duration during night has been related to more impulsive errors on a computerized go/no-go test in 3-5 year old children, indicating poor Inhibitory Control (IC) (Lam et al., 2011). 
Despite evidence to support the foundation of EF during infancy, with many EF skills and maturation of networks related to EF emerging during the first years of life (Diamond, 2006; Grossmann, 2013), studies examining the associations between sleep and EF outcomes in infants and/or toddlers are limited, with most evidence of this association being reported from studies of preschool/school aged children. To our knowledge, only four, mostly small, studies have longitudinally examined the associations between sleep quality and domains of EF in early childhood. In these, (1) parent-reported greater proportion of night sleep at both 12 and 18 months was associated with better IC performance at 26 months of age (Bernier et al., 2010) and at the age of four years (Bernier et al., 2013); (2) actigraphbased lower sleep quality in 12 months-old infants predicted preschoolers' compromised executive attentional control (Sadeh et al., 2015); and (3) infants with high frequency of parent-reported signaled night awakenings at 8 months performed worse in a computerized task of EF, but not in IC or WM tasks, at 24 months of age (Makela et al., 2019).

The importance of further investigating the significance or early sleep is highlighted by the small number of previous studies conducted in young children, the small sample sizes used in these previous studies, the large variability observed in sleep quality and its rapid development in early childhood, and the lack of studies pertaining to multiple forms of sleep disturbances in young children. Furthermore, most of the previous research has reported the effects of disturbed sleep, but very few studies have examined the opposite extreme (i.e., characteristics of "good sleep") and/or the intermediate levels of the sleep distribution. Interestingly, recent studies indicate that sleep duration at both extremes in school-aged children (Chaput et al., 2016) and toddlers (Kocevska et al., 2017) is associated with negative health-related outcomes, while average sleep duration levels are related to better outcomes, indicating the existence of an inverted U-shaped pattern, rather than a linear association. According to this, further studies should consider both the opposite extreme and the intermediate levels of sleep performance, in order to determine whether they contribute differentially to EF. Examining the effects of these three different early sleep categories on EF in infancy/toddlerhood provides a novel understanding of the role of different dimensions of infant sleep on EF development in toddlers.

To address these gaps in the previous literature, the objectives of this study were to investigate in a large sample of young children whether parent-reported sleep quality and/or sleep duration in infants at 6 and 12 months are associated with EF at 30 months, more specifically IC and WM performance. First, based on the existing literature on infants (Bernier et al., 2010), we hypothesized that higher proportion of daytime sleep during the first year of life would be associated with lower IC performance at the age of 30 months. Second, we hypothesized that shorter nighttime sleep is associated with lower performance in both IC and WM tasks, due to the cross-sectional associations between shorter nighttime sleep duration with poorer IC and WM in school-aged children (Cho et al., 2015; Lam et al., 2011). Third, we hypothesized that greater amount of night awakenings would be related to lower IC performance, as higher frequency of night awakenings has been associated with difficulties in behavioral inhibition in school-aged children (Sadeh et al., 2002). Finally, in addition to the children with possible sleep problems (i.e., "the bad sleepers"), we also separately considered the cases that are in the opposite extreme (i.e., "the good sleepers") and who are in between both extremes (i.e., "the intermediate sleepers"). To our knowledge, the relevance of the infants' "good sleep" extreme and the "average sleep" in toddlers' EF performance has not been investigated yet.

\section{Materials And Methods}

\section{Participants}

This study was based on the FinnBrain Birth Cohort Study [www.finnbrain.fi] (Karlsson et al., 2018), which comprises consecutive women at gestational week 12 attending the free-of-charge ultrasounds at Turku University Hospital, in Finland, their children-to-be-born, and fathers of the children/partners of the mothers $(\mathrm{N}=3,808$ mothers and $\mathrm{N}=2,623$ 
fathers). The current study included children whose parents reported the children' sleep at 6 or 12 months and who participated in the development assessment at 30 months of age $(\mathrm{N}=364)$.

\section{Measures}

\section{Key variables}

Sleep questionnaire. Parents' perceptions about their infants sleep and sleep problems at 6 and 12 months was assessed using the Brief Infant Sleep Questionnaire (BISQ), which was filled in by the mothers (Sadeh, 2004). It includes thirteen items that must be completed by the parents and they refer to their perception on their child's sleep during the past week. The BISQ has been validated against actigraphy and sleep diaries and it has demonstrated high test-retest reliability (Sadeh, 2004). Based on previous literature, the variables of interest were: i) nighttime sleep duration; ii) daytime sleep duration; iii) frequency of night awakenings per night; and iv) time awake at night. All these items are open questions. In addition, two additional sleep variables were created for the purpose of this study: vii) total sleep duration per $24 \mathrm{~h}$ (nighttime sleep duration + daytime sleep duration), and viii) proportion of daytime sleep (daytime sleep/total sleep duration per $24 \mathrm{~h} * 100$ ). Finally, all the sleep variables were categorized in three groups, where the cutoffs were set at 10th, 10-90th and 90th percentiles at 6 and 12 months.

Executive functioning (IC and WM). At 30 months, two tasks of EF were used: modified Snack Delay (Kochanska et al., 2000) to measure IC, and Spin the Pots (Hughes \& Ensor, 2005) to measure WM.

In the Snack Delay task, the children were seated at a table and asked to place their hands on a mat depicting the pictures of the hands. The snacks (M\&Ms or raisins according to the parent's choice) were placed under the cup and the child was instructed that when a bell is rung by the experimenter, they are allowed to eat the snack. A total of six trials with delays ranging from $10 \mathrm{~s}$ to $60 \mathrm{~s}$ were conducted and during the trials, the experimenter reached for and picked up the bell without ringing it 1 or 2 times before actually ringing the bell after the specified delay. Scores for each trial range from 0 to 4 ( 0 = "Child eats the snack before the bell was raised, 1 = "Child eats the snack after the bell was raised but before it was rung", 2 = "Child touches the cup or the bell before the bell is raised", $3=$ "Child touches the cup or the bell after the bell is raised", 4 = "Child waits when the bell has rung"). Additionally, child was given up to 2 extra points based on whether he or she was able to keep the hands on the mat during the trials. Maximum score in the task is 36 , higher scores indicating better IC (Spinrad, Eisenberg, \& Gaertner, 2007).

In the Spin the Pots task, six distinct stickers were hidden under eight visually distinct boxes that were laid on a Lazy Susan Tray. In each trial, child was allowed to choose one box and search for the sticker. After each trial, the tray was covered by an opaque scarf and rotated 180 degrees. The task terminated when children found all hidden stickers or when the maximum number of spins was reached (16 spins maximum). Final score was calculated as the number of trials - number of unsuccessful attempts to find a sticker, maximum score being 16 and higher score reflecting better WM performance.

\section{Other measures}

Cognitive functioning at $\mathbf{3 0}$ months. The INTERGROWTH-21st Neurodevelopmental Assessment (INTER-NDA) is a novel, comprehensive assessment of cognition, language, fine and gross motor skills, and behaviour for children aged 22 to 30 months designed for administration in high-, middle- and low-income settings and across populations and languages (Villar et al., 2019). Its 37 items are administered in approximately 15 minutes using a combination of neuropsychological techniques (Fernandes et al., 2014). Children's performance is scored on a 5-point scale, where higher scores reflect better performance for all domains except for negative and global behaviour. The INTER-NDA has been validated against the Bayley Scales of Infant Development - III edition (Murray et al., 2018), and has been shown 
to have good test-retest reliability $(\mathrm{k}=0.79,95 \% \mathrm{Cl}: 0.48-0.96)$ and inter-rater reliability $(\mathrm{k}=0.70,95 \% \mathrm{Cl}: 0.47-0.88)$ (Fernandes et al., 2014). For this specific study, we used the INTER-NDA mean cognitive score in our analysis, as it is more closely related to EF than motor and language domains.

Socio-demographic measures. Additionally, we collected information about the following background variables: i) children's variables: age at the 30-month visit (in days), sex ( $1=$ girls; 2 = boys), gestational age (in weeks), and birth weight (in kg); ii) maternal variables: educational level (i.e. 1 = primary, 2 = secondary, 3 = higher), and maternal age when baby was born (in years), and parity (i.e. 1 = first vs 2 = others). Most of these data were obtained from the Finnish National Register (www.thl.fi) (age, sex, parity, gestational age, birth weight and maternal age when baby was born) or by maternal self-report (level of education).

\section{Procedure}

Altogether, 472 children participated in the developmental assessment of the FinnBrain Child Development and Parental Functioning Lab in the research site of the University of Turku at 30 months of age. The visits in a length of 1.5 hours were carried out by 2 researchers (clinical psychologists and/or advanced psychology students). From all children participating in EF task assessments, 47 (9.96\%) in Snack Delay and 42 (8.90\%) children in Spin the Pots were excluded from the final analyses due to reliability problems of the relevant measurement (e.g. administrator mistake, child is too restless or unable to concentrate). Furthermore, only those children of the remaining 425/430 (90.04/91.10\%) sample whose mothers reported on sleep at 6 and/or12 months were included in the analyses, resulting in the following samples: i) for IC task, 359 infants with sleep questionnaire at 6 months and 322 with sleep questionnaire at 12 months; and ii) for WM task at 30 months, 364 infants with sleep questionnaire at 6 months and 327 with sleep questionnaire at 12 months. A flowchart of the study sampling procedure can be found in Fig. 1.

\section{Statistical analyses}

Statistical analyses were performed with SPSS Statistics V25.0. Descriptive statistics were conducted to obtain means, standard deviations, frequencies and percentages of the variables of interest. Total scores of both EF tasks were negatively skewed. Therefore, we normalized these scores using logarithm transformation. After this, the transformed scores appeared normally distributed, with all skewness values being between -0.5 and 0.5 , indicating that the distribution is approximately symmetric. First, Pearson correlations (for continuous variables) and analysis of variance (ANOVA) test (for categorical and dichotomous variables) between our key variables (sleep and EF measures) and other variables of interest (INTER-NDA cognitive score, child sex, age at 30-months visit and birth weight, gestational age, parity, and maternal education and age when infants was born) were conducted to define potential covariates used in subsequent analyses. Second, to examine the association of sleep with IC (Snack Delay) and WM (Spin the Pots), the sleep variables (nighttime sleep duration, daytime sleep duration, total sleep duration per $24 \mathrm{~h}$, proportion of daytime sleep, number of night awakenings per night, and time awake at night) were categorized in three groups, where the cutoffs were set at 10th, 10th -90th and 90th percentiles at 6 and 12 months.

To compare the EF in these groups, we used ANOVA tests. The first model was an unadjusted model. In the final adjusted model, those covariates that significantly correlated with any of the two outcomes were included (gestational age, maternal education, age at 30 months, child's sex and INTER-NDA mean cognitive score at 30 months). Fisher's Least Significant Difference (LSD) post-hoc test was calculated to evaluate significant group differences.

\section{Results}

\section{Sociodemographic, cognitive and sleep variables}


The sociodemographic, cognitive and sleep variables are presented in Table 1. The frequency distributions of the sleep groups at each time point ( 6 and 12 months) are shown in Table 2. Correlations between the potential covariates with EF and sleep variables appear in Table 3. 
Sociodemographic, cognitive and sleep variables at 6 and 12 months

\section{Sociodemographic variables, categorical}

$$
N(\%)
$$

Gender (girls/boys)

166 (45.9) / 196 (54.1)

Birth order (first/other)

183 (52.1) / 168 (47.9)

Maternal education level, pregnancy (primary/secondary/higher)

$81(23.1) / 113(32.2) / 157(44.7)$

\section{Sociodemographic variables, continuous}

\begin{tabular}{|c|c|c|c|}
\hline & Mean (SD) & Min & $\operatorname{Max}$ \\
\hline Child's age at 30 months of EF assessment, days & $917.13(13.75)$ & 883.00 & 986.00 \\
\hline Gestational age, weeks & $39.81(1.52)$ & 31.86 & 42.43 \\
\hline Maternal age when baby born, years & $31.04(4.28)$ & 19.00 & 42.00 \\
\hline Infant birth weight, kg & $3.57(0.50)$ & 1.47 & 5.47 \\
\hline Infant birth height, cm & $50.62(2.23)$ & 39.00 & 57.00 \\
\hline Number of siblings & $0.74(0.87)$ & 0.00 & 5.00 \\
\hline \multicolumn{4}{|l|}{ Cognitive variables, at 30 months } \\
\hline INTER-NDA Mean Cognitive Score & $3.57(0.32)$ & 1.92 & 4.00 \\
\hline Snack Delay, total score & $27.91(8.27)$ & 0.00 & 36.00 \\
\hline Spin the Pots, total score & $11.99(3.53)$ & 3.00 & 16.00 \\
\hline \multicolumn{4}{|l|}{ Sleep variables at 6 months } \\
\hline BISQ nighttime sleep duration, hours & $9.87(1.17)$ & 1.00 & 12.00 \\
\hline BISQ daytime time sleep duration, hours & $3.82(1.29)$ & 1.50 & 11.00 \\
\hline BISQ total sleep duration, hours & $13.66(1.50)$ & 5.50 & 18.50 \\
\hline BISQ proportion daytime sleep, \% & $27.48(7.66)$ & 12.00 & 81.82 \\
\hline BISQ number of awakenings / night & $2.41(1.60)$ & 0.00 & 11.00 \\
\hline BISQ time awake / night, hours & $0.44(0.44)$ & 0.00 & 2.00 \\
\hline \multicolumn{4}{|l|}{ Sleep variables at 12 months } \\
\hline BISQ nighttime sleep duration, hours & $10.26(0.98)$ & 4.00 & 13.00 \\
\hline BISQ daytime time sleep duration, hours & $2.61(1.17)$ & 1.00 & 11.00 \\
\hline BISQ total sleep duration, hours & $12.75(1.02)$ & 7.00 & 16.00 \\
\hline BISQ proportion daytime sleep, \% & $19.64(5.40)$ & 8.00 & 38.00 \\
\hline BISQ number of awakenings / night & $1.85(1.36)$ & 0.00 & 8.00 \\
\hline
\end{tabular}

INTER-NDA = INTERGROWTH-21st Neurodevelopmental Assessment; BISQ = Brief Infant Sleep Questionnaire; SD = Standard deviation 
Sociodemographic variables, categorical

BISQ time awake / night, hours

$0.32(0.52)$

0.00

5.00

INTER-NDA = INTERGROWTH-21st Neurodevelopmental Assessment; BISQ = Brief Infant Sleep Questionnaire; SD = Standard deviation

Table 2

Frequencies of each sleep category at 6 and 12 months

\begin{tabular}{|c|c|c|c|c|}
\hline & \multicolumn{2}{|l|}{6 months } & \multicolumn{2}{|l|}{12 months } \\
\hline & $\begin{array}{l}\text { Cut-offs 10th, 10-90th, } \\
\text { 90th }\end{array}$ & $N(\%)$ & $\begin{array}{l}\text { Cut-offs 10th, 10-90th, } \\
\text { 90th }\end{array}$ & $N(\%)$ \\
\hline \multirow[t]{3}{*}{ Nighttime sleep duration } & Short $(\leq 8.50 \mathrm{~h})$ & $46(11.4)$ & Short $(<9 \mathrm{~h})$ & $55(15.2)$ \\
\hline & Medium (8.51-11 h) & $\begin{array}{l}256 \\
(63.5)\end{array}$ & Medium (9.01-11) & $\begin{array}{l}186 \\
(51.2)\end{array}$ \\
\hline & Long (> 11 h) & $\begin{array}{l}101 \\
(25.1)\end{array}$ & Long (>11) & $\begin{array}{l}122 \\
(33.6)\end{array}$ \\
\hline \multirow[t]{3}{*}{ Daytime sleep duration } & Short $(\leq 2.25 \mathrm{~h})$ & $61(15)$ & Short $(\leq 1.50 \mathrm{~h})$ & $39(10.6)$ \\
\hline & Medium $(2.26-5.33 \mathrm{~h})$ & $\begin{array}{l}304 \\
(74.9)\end{array}$ & Medium $(1.51-3.50 \mathrm{~h})$ & $\begin{array}{l}271 \\
(73.4)\end{array}$ \\
\hline & Long (> 5.33 h) & $41(10.1)$ & Long $(>3.50 \mathrm{~h})$ & $59(16)$ \\
\hline \multirow[t]{3}{*}{ Total sleep duration per 24 hours } & Short ( $\leq 12 \mathrm{~h})$ & $55(13.7)$ & Short ( $\leq 11 \mathrm{~h})$ & $50(13.9)$ \\
\hline & $\begin{array}{l}\text { Medium (12.01- } \\
15.30 \mathrm{~h})\end{array}$ & $\begin{array}{l}307 \\
(76.4)\end{array}$ & Medium (11.01-14 h) & $\begin{array}{l}254 \\
(70.4)\end{array}$ \\
\hline & Long (> $15.30 \mathrm{~h})$ & $40(10)$ & Long (> $14 \mathrm{~h})$ & $57(15.8)$ \\
\hline \multirow{3}{*}{$\begin{array}{l}\% \text { daytime sleep duration / total } \\
\text { sleep }\end{array}$} & Low $(\leq 18.52 \%)$ & $45(11.2)$ & $\operatorname{Low}(\leq 13 \%)$ & $27(7.5)$ \\
\hline & Medium (18.53-36\%) & $\begin{array}{l}317 \\
(78.9)\end{array}$ & Medium (13.01-27\%) & $296(82)$ \\
\hline & High (> 36\%) & $40(10)$ & High (> 27.01\%) & $38(10.5)$ \\
\hline \multirow[t]{3}{*}{ Frequency of night awakenings } & Low $(\leq 0.5$ times $)$ & $42(10.5)$ & Low ( $\leq$ 0times) & $42(11.8)$ \\
\hline & Medium (0.6-4times) & $\begin{array}{l}311 \\
(77.9)\end{array}$ & Medium (0.1-3.50times) & $\begin{array}{l}271 \\
(76.1)\end{array}$ \\
\hline & High (> 4times) & $46(11.5)$ & High (> 3.50times) & $43(12.1)$ \\
\hline \multirow[t]{3}{*}{ Time awake at night } & Short ( $\leq$ Ohours) & $42(11)$ & Short ( $\leq 0.01$ hours ) & $54(16.4)$ \\
\hline & $\begin{array}{l}\text { Medium (0.01- } \\
0.75 \text { hours) }\end{array}$ & $\begin{array}{l}271 \\
(70.9)\end{array}$ & )$^{\text {Medium }(0.02-0.83 \text { hours }}$ & $\begin{array}{l}241 \\
(73.3)\end{array}$ \\
\hline & Long (>0.75hours) & $69(18.1)$ & Long (>0.83hours ) & $34(10.3)$ \\
\hline
\end{tabular}


Table 3

Correlations between sociodemographic measures, and sleep at 6 and 12 months, and executive function at 30 months

\begin{tabular}{|c|c|c|c|c|c|c|c|c|}
\hline & $\begin{array}{l}\text { Child's } \\
\text { age at } \\
30- \\
\text { month }\end{array}$ & $\begin{array}{l}\text { Child's } \\
\text { sex }\end{array}$ & Parity & $\begin{array}{l}\text { Gestational } \\
\text { age }\end{array}$ & $\begin{array}{l}\text { Birth } \\
\text { weight }\end{array}$ & $\begin{array}{l}\text { INTER- } \\
\text { NDA } \\
\text { mean } \\
\text { cognitive } \\
\text { score }\end{array}$ & $\begin{array}{l}\text { Maternal } \\
\text { educational } \\
\text { level }\end{array}$ & $\begin{array}{l}\text { Maternal } \\
\text { age } \\
\text { when } \\
\text { baby } \\
\text { born }\end{array}$ \\
\hline & $r(p)$ & $F(p)$ & $F(p)$ & $r(p)$ & $r(p)$ & $r(p)$ & $F(p)$ & $r(p)$ \\
\hline $\begin{array}{l}\text { BISQ nighttime } \\
\text { sleep duration at } \\
6 \text { months }\end{array}$ & $\begin{array}{l}0.109 \\
(0.044)\end{array}$ & $\begin{array}{l}0.219 \\
(0.640)\end{array}$ & $\begin{array}{l}0.783 \\
(0.377)\end{array}$ & $\begin{array}{l}0.040 \\
(0.418)\end{array}$ & $\begin{array}{l}-0.009 \\
(0.855)\end{array}$ & $\begin{array}{l}0.021 \\
(0.683)\end{array}$ & $\begin{array}{l}0.676 \\
(0.509)\end{array}$ & $\begin{array}{l}-0.096 \\
(0.054)\end{array}$ \\
\hline $\begin{array}{l}\text { BISQ nighttime } \\
\text { sleep duration at } \\
12 \text { months }\end{array}$ & $\begin{array}{l}-0.063 \\
(0.268)\end{array}$ & $\begin{array}{l}0.102 \\
(0.750)\end{array}$ & $\begin{array}{l}0.271 \\
(0.603)\end{array}$ & $\begin{array}{l}0.123 \\
(0.019)\end{array}$ & $\begin{array}{l}0.034 \\
(0.523)\end{array}$ & $\begin{array}{l}-0.003 \\
(0.948)\end{array}$ & $\begin{array}{l}2.766 \\
(0.064)\end{array}$ & $\begin{array}{l}0.128 \\
(0.015)\end{array}$ \\
\hline $\begin{array}{l}\text { BISQ daytime } \\
\text { time sleep } \\
\text { duration at } 6 \\
\text { months }\end{array}$ & $\begin{array}{l}0.040 \\
(0.462)\end{array}$ & $\begin{array}{l}1.561 \\
(0.212)\end{array}$ & $\begin{array}{l}8.418 \\
(0.004)\end{array}$ & $\begin{array}{l}-0.028 \\
(0.578)\end{array}$ & $\begin{array}{l}0.095 \\
(0.058)\end{array}$ & $\begin{array}{l}-0.028 \\
(0.581)\end{array}$ & $\begin{array}{l}0.034 \\
(0.966)\end{array}$ & $\begin{array}{l}0.042 \\
(0.398)\end{array}$ \\
\hline $\begin{array}{l}\text { BISQ daytime } \\
\text { time sleep } \\
\text { duration at } 12 \\
\text { months }\end{array}$ & $\begin{array}{l}0.011 \\
(0.849)\end{array}$ & $\begin{array}{l}1.057 \\
(0.305)\end{array}$ & $\begin{array}{l}0.009 \\
(0.923)\end{array}$ & $\begin{array}{l}0.035 \\
(0.499)\end{array}$ & $\begin{array}{l}0.022 \\
(0.673)\end{array}$ & $\begin{array}{l}-0.018 \\
(0.730)\end{array}$ & $\begin{array}{l}0.176 \\
(0.839)\end{array}$ & $\begin{array}{l}0.028 \\
(0.591)\end{array}$ \\
\hline $\begin{array}{l}\text { BISQ total sleep } \\
\text { duration at } 6 \\
\text { months }\end{array}$ & $\begin{array}{l}0.100 \\
(0.064)\end{array}$ & $\begin{array}{l}0.004 \\
(0.947)\end{array}$ & $\begin{array}{l}9.380 \\
(0.002)\end{array}$ & $\begin{array}{l}0.015 \\
(0.768)\end{array}$ & $\begin{array}{l}0.095 \\
(0.059)\end{array}$ & $\begin{array}{l}-0.027 \\
(0.589)\end{array}$ & $\begin{array}{l}0.705 \\
(0.495)\end{array}$ & $\begin{array}{l}-0.082 \\
(0.100)\end{array}$ \\
\hline $\begin{array}{l}\text { BISQ total sleep } \\
\text { duration at } 12 \\
\text { months }\end{array}$ & $\begin{array}{l}-0.089 \\
(0.119)\end{array}$ & $\begin{array}{l}2.843 \\
(0.093)\end{array}$ & $\begin{array}{l}0.000 \\
(0.990)\end{array}$ & $\begin{array}{l}0.142 \\
(0.007)\end{array}$ & $\begin{array}{l}0.095 \\
(0.072)\end{array}$ & $\begin{array}{l}-0.044 \\
(0.402)\end{array}$ & $\begin{array}{l}3.224 \\
(0.041)\end{array}$ & $\begin{array}{l}-0.104 \\
(0.049)\end{array}$ \\
\hline $\begin{array}{l}\text { BISQ proportion } \\
\text { daytime sleep at } \\
6 \text { months }\end{array}$ & $\begin{array}{l}-0.038 \\
(0.479)\end{array}$ & $\begin{array}{l}0.844 \\
(0.359)\end{array}$ & $\begin{array}{l}3.550 \\
(0.060)\end{array}$ & $\begin{array}{l}-0.040 \\
(0.429)\end{array}$ & $\begin{array}{l}0.094 \\
(0.060)\end{array}$ & $\begin{array}{l}-0.042 \\
(0.405)\end{array}$ & $\begin{array}{l}0.136 \\
(0.873)\end{array}$ & $\begin{array}{l}0.020 \\
(0.692)\end{array}$ \\
\hline $\begin{array}{l}\text { BISQ proportion } \\
\text { daytime sleep at } \\
12 \text { months }\end{array}$ & $\begin{array}{l}0.028 \\
(0.627)\end{array}$ & $\begin{array}{l}1.423 \\
(0.234)\end{array}$ & $\begin{array}{l}0.309 \\
(0.579)\end{array}$ & $\begin{array}{l}-0.041 \\
(0.433)\end{array}$ & $\begin{array}{l}0.053 \\
(0.319)\end{array}$ & $\begin{array}{l}-0.039 \\
(0.464)\end{array}$ & $\begin{array}{l}0.275 \\
(0.760)\end{array}$ & $\begin{array}{l}0.055 \\
(0.198)\end{array}$ \\
\hline $\begin{array}{l}\text { BISQ number of } \\
\text { awakenings/night } \\
\text { at } 6 \text { months }\end{array}$ & $\begin{array}{l}0.058 \\
(0.284)\end{array}$ & $\begin{array}{l}4.474 \\
(0.035)\end{array}$ & $\begin{array}{l}0.012 \\
(0.913)\end{array}$ & $\begin{array}{l}-0.071 \\
(0.156)\end{array}$ & $\begin{array}{l}-0.084 \\
(0.096)\end{array}$ & $\begin{array}{l}-0.008 \\
(0.877)\end{array}$ & $\begin{array}{l}2.017 \\
(0.134)\end{array}$ & $\begin{array}{l}0.035 \\
(0.481)\end{array}$ \\
\hline $\begin{array}{l}\text { BISQ number of } \\
\text { awakenings/night } \\
\text { at } 12 \text { months }\end{array}$ & $\begin{array}{l}-0.002 \\
(0.975)\end{array}$ & $\begin{array}{l}0.532 \\
(0.466)\end{array}$ & $\begin{array}{l}0.078 \\
(0.780)\end{array}$ & $\begin{array}{l}-0.133 \\
(0.012)\end{array}$ & $\begin{array}{l}-0.079 \\
(0.138)\end{array}$ & $\begin{array}{l}-0.072 \\
(0.179)\end{array}$ & $\begin{array}{l}2.952 \\
(0.054)\end{array}$ & $\begin{array}{l}0.153 \\
(0.004)\end{array}$ \\
\hline $\begin{array}{l}\text { BISQ time awake } \\
\text { / night at } 6 \\
\text { months }\end{array}$ & $\begin{array}{l}0.158 \\
(0.004)\end{array}$ & $\begin{array}{l}0.577 \\
(0.448)\end{array}$ & $\begin{array}{l}1.245 \\
(0.265)\end{array}$ & $\begin{array}{l}-0.028 \\
(0.580)\end{array}$ & $\begin{array}{l}-0.004 \\
(0.941)\end{array}$ & $\begin{array}{l}-0.039 \\
(0.454)\end{array}$ & $\begin{array}{l}0.785 \\
(0.457)\end{array}$ & $\begin{array}{l}0.148 \\
(0.004)\end{array}$ \\
\hline $\begin{array}{l}\text { BISQ time awake } \\
\text { / night at } 12 \\
\text { months }\end{array}$ & $\begin{array}{l}0.019 \\
(0.748)\end{array}$ & $\begin{array}{l}0.024 \\
(0.876)\end{array}$ & $\begin{array}{l}0.297 \\
(0.586)\end{array}$ & $\begin{array}{l}-0.046 \\
(0.406)\end{array}$ & $\begin{array}{l}-0.046 \\
(0.246)\end{array}$ & $\begin{array}{l}0.000 \\
(0.995)\end{array}$ & $\begin{array}{l}1.287 \\
(0.278)\end{array}$ & $\begin{array}{l}0.038 \\
(0.493)\end{array}$ \\
\hline
\end{tabular}

*For the correlation analyses, the continuous values of each of the sleep variables were used (i.e., total score).

Pearson correlations ( $r$ ) were used for all the covariates, except for maternal educational level, child's sex and parity where ANOVA test (F) was applied.

${ }^{* *}$ Correlation between Snack Delay, Hands version and Spin the Pots: $r=0.239, p<0.001$ 


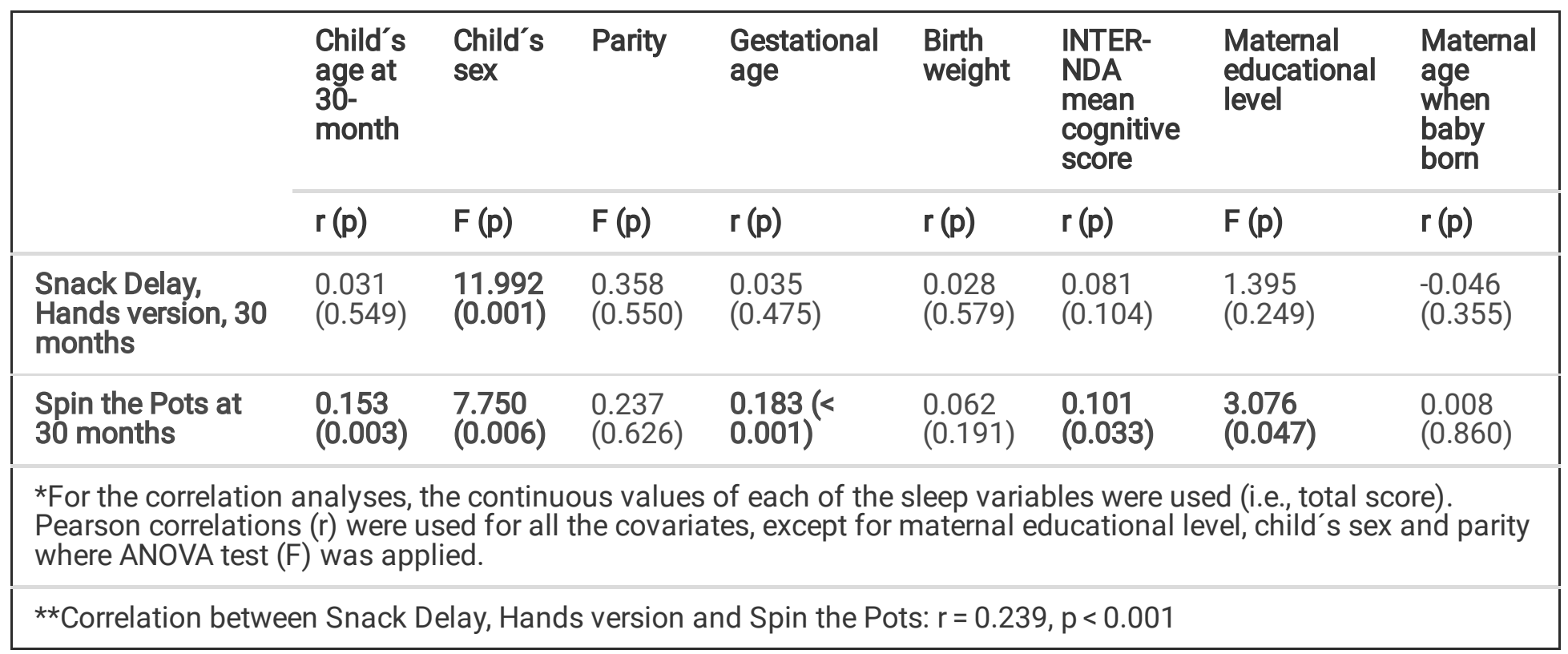

\section{Sleep at 6 and 12 months and IC at $\mathbf{3 0}$ months}

In the 1st ANOVA, we did not find any significant associations between sleep at 6 and 12 months and IC at 30 months, although the proportion of daytime sleep at both 6 and 12 months was an almost significant predictor of IC $[F(2,317)=$ 2.614, $p=0.075, \nabla^{2}=0.016$; and $F(2,280)=3.003, p=0.051, \nabla^{2}=0.021$, respectively]. However, when we controlled for cognitive level and other potential confounding factors in the adjusted model, proportion of daytime sleep at 12 months reached the statistical significance $\left[F(7,271)=3.012, p=0.039, \eta^{2}=0.022\right]$. More specifically, the post-hoc tests showed that 12-month-old infants with both greater (90th percentile) and smaller proportion of daytime sleep (10th percentile) had worse IC functioning at 30 months than infants with average proportion of daytime sleep at the age of 12 months (10th -90 th percentile; $p=0.020$ and $p=0.030$, respectively). See Table 4 for all statistical values in IC. 
Table 4

The differences between sleep category at 6 months and 12 months in predicting child inhibitory control at 30 months 6 months

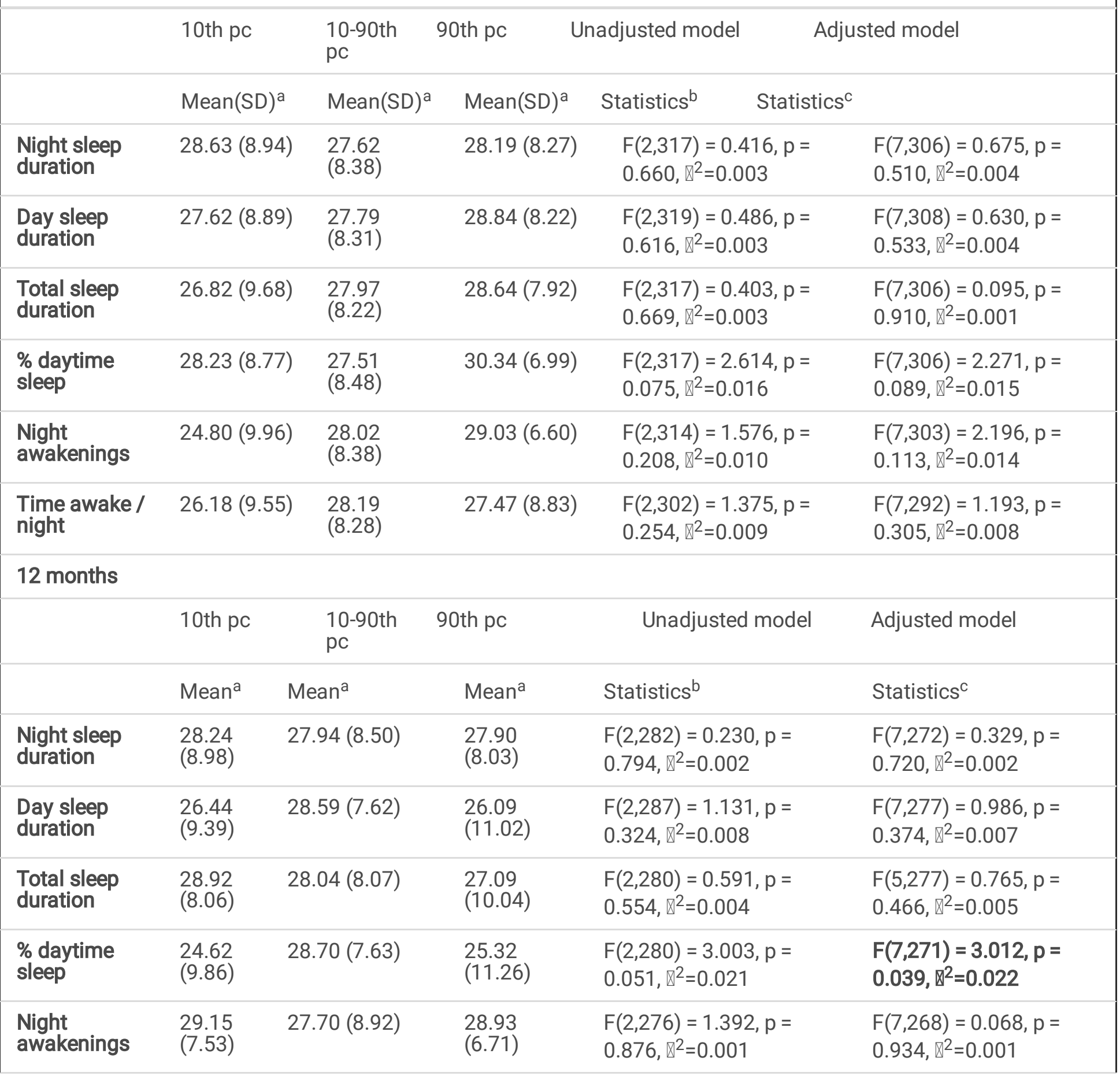

a Mean and standard deviation (SD) of each group, in each of the sleep variables, are obtained from the raw outcome measure

bUnadjusted model: No covariates

${ }^{c}$ Adjusted model: gestational age, maternal education, age at 30 months, child's sex and INTER-NDA mean cognitive score at 30 months

*Similar associations were obtained with non-transformed outcomes 


\begin{tabular}{|c|c|c|c|c|c|}
\hline \multicolumn{6}{|l|}{6 months } \\
\hline $\begin{array}{l}\text { Time awake / } \\
\text { night }\end{array}$ & $\begin{array}{l}29.42 \\
(7.36)\end{array}$ & $20.07(0.03)$ & (7.14) & $\begin{array}{l}F(2,253)=0.443, p= \\
0.643, \rrbracket^{2}=0.003\end{array}$ & $\begin{array}{l}F(7,245)=0.294, p= \\
0.746 \rrbracket^{2}=0.002\end{array}$ \\
\hline
\end{tabular}

aMean and standard deviation (SD) of each group, in each of the sleep variables, are obtained from the raw outcome measure

bUnadjusted model: No covariates

${ }^{c}$ Adjusted model: gestational age, maternal education, age at 30 months, child's sex and INTER-NDA mean cognitive score at 30 months

*Similar associations were obtained with non-transformed outcomes

\section{Sleep at six and 12 months and WM at 30 months}

In the 1st ANOVA, where no covariates were included, we found significant differences in WM according to nighttime sleep duration at 6 months $\left[F(2,347)=3.626, p=0.028, \eta^{2}=0.020\right]$. The post-hoc test showed that infants with longer sleep duration during nighttime (90th percentile) performed better than the infants with short sleep duration during nighttime ( $\leq 10$ th percentile; $p=0.015$ ) and infants with normal ranges of nighttime sleep duration (10th -90th percentile; $p=0.036)$. However, this association did not remain significant when we controlled for the covariates (Table 5). 
Table 5

The differences between sleep category at 6 months and 12 months in predicting child working memory at 30 months

6 months

\begin{tabular}{|c|c|c|c|c|c|}
\hline & 10th pc & 10-90th & 90th pc & Unadjusted model & Adjusted model \\
\hline & Mean(SD) ${ }^{a}$ & $\operatorname{Mean}(S D)^{a}$ & $\operatorname{Mean}(S D)^{a}$ & Statistics ${ }^{b}$ & Statistics $^{c}$ \\
\hline $\begin{array}{l}\text { Night sleep } \\
\text { duration }\end{array}$ & $11.15(4.12)$ & $\begin{array}{l}11.81 \\
(3.39)\end{array}$ & 12.39 (3.66) & $\begin{array}{l}F(2,347)=3.626, p= \\
0.028, \otimes 2=0.020\end{array}$ & $\begin{array}{l}F(7,334)=2.019, p= \\
0.134, \nabla^{2}=0.012\end{array}$ \\
\hline $\begin{array}{l}\text { Day sleep } \\
\text { duration }\end{array}$ & $12.58(3.18)$ & $\begin{array}{l}11.83 \\
(3.59)\end{array}$ & $11.06(3.78)$ & $\begin{array}{l}F(2,348)=1.747 p= \\
0.176, \otimes 2=0.010\end{array}$ & $\begin{array}{l}F(7,335)=1.495, p= \\
0.226, \nabla^{2}=0.009\end{array}$ \\
\hline $\begin{array}{l}\text { Total sleep } \\
\text { duration }\end{array}$ & $12.00(3.66)$ & $\begin{array}{l}11.90 \\
(3.55)\end{array}$ & 11.75 (3.47) & $\begin{array}{l}F(2,346)=0.068, p= \\
0.934 \varangle 2=0.000\end{array}$ & $\begin{array}{l}F(7,333)=0.298, p= \\
0.742, \nabla^{2}=0.002\end{array}$ \\
\hline $\begin{array}{l}\text { \% daytime } \\
\text { sleep }\end{array}$ & $12.33(3.60)$ & $\begin{array}{l}11.94 \\
(3.46)\end{array}$ & $11.06(4.11)$ & $\begin{array}{l}F(2,346)=1.142, p= \\
0.320, \otimes 2=0.007\end{array}$ & $\begin{array}{l}F(7,333)=0.822, p= \\
0.440, \nabla^{2}=0.005\end{array}$ \\
\hline $\begin{array}{l}\text { Night } \\
\text { awakenings }\end{array}$ & 10.91 (4.09) & $\begin{array}{l}11.91 \\
(3.58)\end{array}$ & $12.33(2.82)$ & $\begin{array}{l}F(2,342)=0.477, p= \\
0.621, \otimes 2=0.003\end{array}$ & $\begin{array}{l}F(7,329)=0.963, p= \\
0.383, \nabla^{2}=0.006\end{array}$ \\
\hline $\begin{array}{l}\text { Time awake / } \\
\text { night }\end{array}$ & 11.22 (3.50) & $\begin{array}{l}12.28 \\
(3.23)\end{array}$ & $11.12(4.19)$ & $\begin{array}{l}F(2,332)=1.534, p= \\
0.217, \otimes 2=0.009\end{array}$ & $\begin{array}{l}F(7,320)=1.879, p= \\
0.154, \nabla^{2}=0.012\end{array}$ \\
\hline \multicolumn{6}{|l|}{12 months } \\
\hline & 10th pc & $\begin{array}{l}10-90 \text { th } \\
\text { pc }\end{array}$ & 90th pc & Unadjusted model & Adjusted model \\
\hline & Mean $^{a}$ & Mean $^{a}$ & Mean $^{a}$ & Statistics $^{b}$ & Statistics ${ }^{c}$ \\
\hline $\begin{array}{l}\text { Night sleep } \\
\text { duration }\end{array}$ & $\begin{array}{l}11.39 \\
(4.03)\end{array}$ & 12.30 (3.22) & $\begin{array}{l}11.70 \\
(3.57)\end{array}$ & $\begin{array}{l}F(2,310)=0.572, p= \\
0.565, \rrbracket^{2}=0.004\end{array}$ & $\begin{array}{l}F(7,298)=0.972, p= \\
0.379, \rrbracket^{2}=0.006\end{array}$ \\
\hline $\begin{array}{l}\text { Day sleep } \\
\text { duration }\end{array}$ & $\begin{array}{l}11.71 \\
(3.87)\end{array}$ & $12.16(3.46)$ & $\begin{array}{l}11.31 \\
(3.98)\end{array}$ & $\begin{array}{l}F(2,316)=1.113, p= \\
0.330, \rrbracket^{2}=0.007\end{array}$ & $\begin{array}{l}F(7,304)=1.123, p= \\
0.327, \rrbracket^{2}=0.007\end{array}$ \\
\hline $\begin{array}{l}\text { Total sleep } \\
\text { duration }\end{array}$ & $\begin{array}{l}12.29 \\
(3.58)\end{array}$ & $12.02(3.56)$ & $\begin{array}{l}11.33 \\
(3.70)\end{array}$ & $\begin{array}{l}F(2,308)=0.898, p= \\
0.409, \nabla^{2}=0.006\end{array}$ & $\begin{array}{l}F(7,297)=2.020, p= \\
0.135, \rrbracket^{2}=0.013\end{array}$ \\
\hline $\begin{array}{l}\% \text { daytime } \\
\text { sleep }\end{array}$ & $\begin{array}{l}11.69 \\
(3.72)\end{array}$ & $12.12(3.49)$ & $\begin{array}{l}10.88 \\
(4.07)\end{array}$ & $\begin{array}{l}F(2,308)=1.565, p= \\
0.211, \nabla^{2}=0.010\end{array}$ & $\begin{array}{l}F(7,297)=2.075, p= \\
0.127, \rrbracket^{2}=0.014\end{array}$ \\
\hline $\begin{array}{l}\text { Night } \\
\text { awakenings }\end{array}$ & $\begin{array}{l}12.82 \\
(3.24)\end{array}$ & 11.91 (3.69) & $\begin{array}{l}11.61 \\
(3.08)\end{array}$ & $\begin{array}{l}F(2,304)=2.038, p= \\
0.132, \nabla^{2}=0.013\end{array}$ & $\begin{array}{l}F(7,294)=1.283, p= \\
0.279, \nabla^{2}=0.009\end{array}$ \\
\hline
\end{tabular}

aMean and standard deviation (SD) of each group, in each of the sleep variables, are obtained from the raw outcome measure

bUnadjusted model: No covariates

${ }^{\mathrm{c}}$ Adjusted model: gestational age, maternal education, age at 30 months, child's sex and INTER-NDA mean cognitive score at 30 months

*Similar associations were obtained with non-transformed outcomes 


\section{6 months}

\begin{tabular}{|c|c|c|c|c|c|}
\hline $\begin{array}{l}\text { Time awake / } \\
\text { night }\end{array}$ & $\begin{array}{l}12.91 \\
(3.05)\end{array}$ & $12.00(3.60)$ & $\begin{array}{l}10.32 \\
(3.82)\end{array}$ & $\begin{array}{l}F(2,282)=5.002, p= \\
0.007, \rrbracket^{2}=0.034\end{array}$ & $\begin{array}{l}F(7,271)=4.731, p= \\
0.014, \rrbracket^{2}=0.031\end{array}$ \\
\hline
\end{tabular}

aMean and standard deviation (SD) of each group, in each of the sleep variables, are obtained from the raw outcome measure

bUnadjusted model: No covariates

cAdjusted model: gestational age, maternal education, age at 30 months, child's sex and INTER-NDA mean cognitive score at 30 months

*Similar associations were obtained with non-transformed outcomes

In addition, time awake during night at 12 months was associated with WM performance $[F(2,282)=5.002, p=0.007$, $\left.\eta^{2}=0.034\right]$. The post-hoc test showed that 12-month-old infants who spent long time awake during night ( $\geq 90$ th percentile) performed worse in the WM task than 12-month-old infants that slept through the night ( $\leq 10$ th percentile; $p$ $=0.002$ ) and also than those infants who spent average time awake during night (10th -90th percentile; $p=0.011$ ). In the adjusted model (Table $4 \mathrm{~b}$ ), we found that this association remained significant when controlling for the covariates $\left[F(7,271)=4.731, p=0.014, \eta^{2}=0.031\right]$. The group differences also remained similar in the post-hoc tests [i.e. the infants who spent less time awake ( $\leq 10$ th percentile) had better performance in WM tasks than infants who spent more time awake ( $\geq 90$ th percentile): $p=0.004$; or 10th -90 th percentile: $p=0.014$ ].

The estimated marginal means for WM and IC at 30 months and for each sleep group at 6 and 12 months are displayed in Fig. 2 (IC) and Fig. 3 (WM).

Interestingly, ANOVA tests were also conducted with non-transformed outcomes, and similar results were observed. For this reason, and for the purpose of this study, we report only here the results with the transformed outcomes.

\section{Discussion}

In this longitudinal birth cohort study, we show that (1) the proportion of daytime sleep at 12 months and IC at 30 months follows an inverted U-shaped relation, and (2) the association between time spent awake during night at 12 months and WM at 30 months is linear. By longitudinally examining the associations between infant sleep and toddler EF across the range of infant sleep outcomes, i.e. not only in those infants with sleep problems but also on those who might be labelled as "good sleepers" and "intermediate sleepers", these findings extend the current understanding of the relationship between infant sleep and toddler EF and provide novel evidence to support a dose-dependent curvilinear relationship between sleep and EF during early childhood.

Consistent with our first hypothesis and with previous longitudinal study in infants (Bernier et al., 2010), we found that proportion of daytime sleep at 12 months was associated with IC at 30 months. While our finding of the inverted-Ushaped association between proportion of daytime sleep and IC is novel, previous cross-sectional studies with toddlers (Kocevska et al., 2017), children (Chaput et al., 2016), and adults (Leng et al., 2015) have suggested that sleep duration at both extremes is associated with negative health-related outcomes. This finding is also consistent with evidence from neuroendocrine literature, which reports inverted U-shaped associations between cortisol levels and cognition in both children (Jager et al., 2014) and adults (Schilling et al., 2013). Taken together, these findings and ours, suggest that non-linear patterns of association may describe the relationship between complex biological processes during early childhood more accurately than linear associations. Furthermore, daytime sleep time seems to be mostly 
determined by maturation (i.e. age) (Paavonen et al., 2020; Weissbluth, 1995), and most of the infants sleep an average of two hours during daytime. Interestingly, one recent longitudinal study reported that inappropriate amounts of daytime sleep were related to worse quality of night-time sleep in three to eight-month-old infants (Paavonen et al., 2019). Therefore, it is likely that infants with average amounts of daytime sleep most likely represent the normal ranges of the developmental stage also in other areas of development, such as cognition and/or self-regulation.

We did not find evidence to support our second hypothesis that shorter nighttime sleep is associated with lower performance in both IC and WM tasks. Such associations have been reported in two cross-sectional studies conducted in school-aged children (Cho et al., 2015; Lam et al., 2011). Our failure to confirm these previous findings could be explained by the fact that significant sleep deprivation might be quite uncommon in infants among whom sleep is strongly driven by homeostatic pressure (Jenni \& LeBourgeois, 2006). Finally, we should take into account that some research supports the notion that EF in early childhood may be best described by a single factor, rather than by different aspects (Espy et al., 2011; Shing et al., 2010; Wiebe, Espy, \& Charak, 2008), due to the fact that EF undergoes rapid development in infancy and that the subdomains of EF are highly interrelated at these early stages (Diamond, 2013).

Concerning our third hypothesis, this was partially confirmed as although we did not find significant associations between the number of night awakenings and IC at 30 months, we did found that time spent awake during the night at 12 months was longitudinally associated with WM performance at 30 months in a linear manner. This suggests that night awakenings are often normative in infants' development, while long periods of time spent awake at night more likely indicates a deviance in sleep quality in early childhood. Number of night awakenings tends to remain stable during the first year of life, ranging from 0 to 3.4 episodes per night for very young infants ( $0-2$ months), to $0-2.5$ per night at the age of 12-24 months (Galland et al., 2012). Therefore, time spent awake at night might a better indicator of disturbed sleep than number of night awakenings, and thus time spent awake at night could be more harmful for the development of some EF, such as WM. Further studies on the effects of sleep fragmentation (i.e., frequency of night awakening and time awake at night) on the development of EF deficits are still needed. To the best of our knowledge, our study is the first one reporting an association between parent-reported time spent awake at night and EF in this age group.

Finally, it should be noted that the lack of association between number of night awakenings and EF could be also related to the use of parent-reported sleep measures in this study. For instance, while infants may briefly wake up during the night, many of them are able to fall back to sleep by themselves, and thus especially the very short awakenings are not necessarily noticed by their parents (Minde et al., 1993). Therefore, the use of more objective sleep measures, such as actigraphy, may be useful to measure the exact frequency of night awakenings. Furthermore, night awakenings are more frequent and more normative in infancy than later during development and, hence, they may have distinct impact on IC performance in older children.

Interestingly, our findings concerning sleep during the first year of life and EF (i.e., IC and WM) at the age of 30 months were only found when sleep was measured at the age of 12 months, while there were no associations between sleep at 6 months and EF at 30 months. One possible explanation could be that the high inter-individual variability in sleep quality which is mainly seen during the first 6 months of life, could be related to environmental factors that temporarily impair sleep in infants (Ednick et al., 2009). Therefore, the effects that sleep at 6 months exerts on later development in toddlers might be less robust. However, recent findings from our group using a different sample showed that parental reported short sleep duration at 3,8 , and 18 months was longitudinally associated with attention difficulties at the age of 5 years (Huhdanpaa et al., 2019). Nevertheless, the cognitive measures used in this previous study and our current study were different (i.e., parent-reported versus behavioral cognitive measures, respectively), and thus the results are not directly comparable 
Overall, our findings support the hypothesis that sleep disruption in early childhood is longitudinally associated with later EF and that different sleep patterns in 12-month-old infants affect distinct aspects of EF (i.e., IC and WM) at the age of 30 months. Considering that EF and its associated neural circuitry experience rapid development during the ages of 2 and 5 years (Best \& Miller, 2010), and that sleep plays a vital restorative role in brain functioning (Medic et al., 2017), disrupted sleep early in development could have negative longitudinal consequences for the development of EF.

The findings of our study suggest that variation in sleep quality more clearly influences the variation in EF when sleep is measured at the infant age of 12 months compared to 6 months. At the age of 12 months, the most relevant sleep quality patterns from the perspective of toddler's WM and IC are the measures related to the acquisition of the circadian rhythm (i.e. proportion of daytime sleep and time awake at night).

The main strength of our study is the large sample size and the longitudinal design, which captures the long-term consequences of early childhood sleep disturbances on IC and WM in toddlers. Moreover, we measured sleep at 6 and 12 months, which enabled us to examine the effects of sleep in very early stages of life. Furthermore, the study is population-based, and we were able to account for various confounding variables, including maternal factors and child cognitive development at 30 months. Another major strength of this study is the approach of using three different sleep groups of "good sleepers", "intermediate sleepers" and "bad sleepers" to study the non-linear associations between sleep and EF.

Our study has some limitations. First, sleep measures were only reported using parental reports, and we did not use objective measures, such as actigraphy. While parental reports and objective reports may disagree in some cases (Molfese et al., 2015), sleep reports are still considered valid for assessing sleep in young children. Moreover, use of parental reports enables the collection of larger samples. Second, our sample was composed of relatively healthy mothers and infants; thus, generalization of the results should be made cautiously with respect to clinical populations. Third, no adjustment for current sleep at 30 months was available in this study, which may result in less accurate findings.

\section{Conclusions}

The main findings of our study show that the association between proportion of daytime sleep at 12 months and IC at 30 months follows an inverted U-shape, while a linear relation between time awake during night at 12 months and WM at 30 months was found. However, no significant associations between sleep at 6 months and any of the EF measures at 30 months were found, reflecting the high inter-individual variability in sleep development occurring at this early stage of life. According to our results, it seems that different sleep difficulties at 12 months affect different aspects of EF (i.e., IC and WM) at 30 months, and also these associations follow different patterns. Further studies should include the measure of time awake at night in addition of night awakening frequency to have a better understanding on the effects that fragmented sleep might have for the development of WM in later stages of life. Finally, rather than assuming that only longer proportion of daytime sleep has adverse effects on IC, daytime sleep proportion at both extremes and also the intermediate levels should be considered in future studies of EF development. If toddlers' EF aspects, such as IC and WM are improved by treating specific sleep difficulties early in infancy, this would be highly relevant for the improvement of children's cognitive functioning.

\section{Abbreviations}

ANOVA: Analysis of variance.

BISQ: Brief Infant Sleep Questionnaire. 

EF: Executive functioning.
IC: Inhibitory control.

INTER-NDA: INTERGROWTH-21st Neurodevelopment Assessment.

WM: Working memory.

\section{Declarations}

\section{Acknowledgments}

Not applicable.

\section{Author contributions}

IMM conceptualized, analyzed and wrote the original draft of the manuscript. SN conducted the behavioural testing, and assisted in the data analyses. TM assisted in the data analyses. EE conducted the nehavioural testing. RR and MF conceptualized the study. HK conceptualized the study and provided funding. EJP conceptualized and supervised the study. LK conceptualized, supervised and provided funding for the study. All authors read, reviewed, edited and approved the final manuscript.

\section{Funding}

This work was supported by The Academy of Finland (grant numbers 134950, 253270, 308589, 308588, and 315035), Finnish State Grants for Clinical Research, Signe and Ane Gyllenberg Foundation, Yrjö Jahnsson Foundation, Alexander von Humboldt Foundation, and Emil Aaltonen Foundation.

\section{Availability of data and materials}

The datasets used and/or analysed during the current study are available from the corresponding author on reasonable request.

\section{Ethics approval and consent to participate}

The parents gave written informed consent on their own and on their child's behalf. The study was approved by the Ethical Committee of the Southwestern Finland Hospital District (number 57/180/2011).

\section{Consent for publication}

Not applicable.

\section{Competing interests}

The authors declare that they have no competing interests. 


\section{References}

1. Astill RG, Van der Heijden KB, Van ljzendoorn MH, Van Someren EJW. Sleep, cognition, and behavioral problems in school-age children: a century of research meta-analyzed. Psychol Bull. 2012; 138(6): 1109-1138. https://doi.org/10.1037/a0028204

2. Bernier A, Carlson SM, Bordeleau S, Carrier J. Relations between physiological and cognitive regulatory systems: infant sleep regulation and subsequent executive functioning. Child Dev. 2010; 81(6): 1739-1752. https://doi.org/10.1111/j.1467-8624.2010.01507.x

3. Best JR, Miller PH. A developmental perspective on executive function. Child Dev. 2010; 81(6): 1641-1660. https://doi.org/10.1111/j.1467-8624.2010.01499.x

4. Cajochen C, Knoblauch V, Krauchi K, Renz C, Wirz-Justice A. Dynamics of frontal EEG activity, sleepiness and body temperature under high and low sleep pressure. Neuroreport. 2011; 12(10): 2277-2281. https://doi.org/10.1097/00001756-200107200-00046

5. Chaput J-P, Gray CE, Poitras VJ, Carson V, Gruber R, Olds T, Tremblay MS. Systematic review of the relationships between sleep duration and health indicators in school-aged children and youth. Appl Physiol Nutr Metab. 2016; 41(6 Suppl 3): S266-82. https://doi.org/10.1139/apnm-2015-0627

6. Cho M, Quach J, Anderson P, Mensah F, Wake M, Roberts G. Poor sleep and lower working memory in grade 1 children: cross-sectional, population-based study. Acad Pediatr. 2015; 15(1): 111-116.

https://doi.org/10.1016/j.acap.2014.06.021

7. Choe M-S, Ortiz-Mantilla S, Makris N, Gregas M, Bacic J, Haehn D, Grant PE. Regional infant brain development: an MRI-based morphometric analysis in 3 to 13 month olds. Cereb Cortex. 2013; 23(9): 2100-2117. https://doi.org/10.1093/cercor/bhs197

8. Dahl RE. The impact of inadequate sleep on children's daytime cognitive function. Semin Pediatr Neurol. 1996; 3(1): 44-50.

9. de Weerd AW, van den Bossche RAS. The development of sleep during the first months of life. Sleep Med Rev. 2003; 7(2): 179-191.

10. Diamond A. Early Development of Executive Functions. Lifespan Cognition: Mechanisms of Change, 2006; 70-95. https://doi.org/10.1093/acprof:oso/9780195169539.003.0006

11. Diamond A (2013). Executive functions. Annu Rev Psychol. 2013; 64: 135-168. https://doi.org/10.1146/annurevpsych-113011-143750

12. Ednick M, Cohen AP, McPhail GL, Beebe D, Simakajornboon N, Amin RS. A review of the effects of sleep during the first year of life on cognitive, psychomotor, and temperament development. Sleep. 2009; 32(11): 1449-1458.

13. Espy KA, Sheffield TD, Wiebe SA, Clark CAC, Moehr MJ. Executive control and dimensions of problem behaviors in preschool children. J Child Psychol Psychiatry. 2011; 52(1): 33-46. https://doi.org/10.1111/j.14697610.2010.02265.x

14. Fernandes M, Stein A, Newton CR, Cheikh-Ismail L, Kihara M, Wulff K, Villar J. The INTERGROWTH-21st Project Neurodevelopment Package: a novel method for the multi-dimensional assessment of neurodevelopment in preschool age children. PloS One. 2014; 9(11): e113360. https://doi.org/10.1371/journal.pone.0113360

15. Finelli LA, Baumann H, Borbely AA, Achermann P. Dual electroencephalogram markers of human sleep homeostasis: correlation between theta activity in waking and slow-wave activity in sleep. Neuroscience. 2000: 101(3): 523-529. https://doi.org/10.1016/s0306-4522(00)00409-7

16. Galland BC, Taylor BJ, Elder DE, Herbison P. Normal sleep patterns in infants and children: a systematic review of observational studies. Sleep Med Rev. 2012; 16(3): 213-222. https://doi.org/10.1016/j.smrv.2011.06.001 
17. Grossmann T. Mapping prefrontal cortex functions in human infancy. Infancy. 2013; 18(3): 303-324. https://doi.org/10.1111/infa.12016

18. Henderson JMT, France KG, Blampied NM. The consolidation of infants' nocturnal sleep across the first year of life. Sleep Med Rev. 2011; 15(4): 211-220. https://doi.org/10.1016/j.smrv.2010.08.003

19. Hughes C, Ensor R. Executive function and theory of mind in 2 year olds: a family affair? Dev Neuropsychol. 2005; 28(2): 645-668. https://doi.org/10.1207/s15326942dn2802_5

20. Huhdanpaa H, Morales-Munoz I, Aronen ET, Polkki P, Saarenpaa-Heikkila O, Paunio T, Paavonen EJ. Sleep Difficulties in Infancy Are Associated with Symptoms of Inattention and Hyperactivity at the Age of 5 Years: A Longitudinal Study. J Dev Behav Pediatr. 2019; 40(6): 432-440. https://doi.org/10.1097/DBP.0000000000000684

21. Jager K, Schmidt M, Conzelmann A, Roebers CM. Cognitive and physiological effects of an acute physical activity intervention in elementary school children. Front Psychol. 2014; 5: 1473.

https://doi.org/10.3389/fpsyg.2014.01473

22. Jenni OG, LeBourgeois MK. Understanding sleep-wake behavior and sleep disorders in children: the value of a model. Curr Opin Psychiatr y. 2006; 19(3): 282-287. https://doi.org/10.1097/01.yco.0000218599.32969.03

23. Karlsson L, Tolvanen M, Scheinin NM, Uusitupa H-M, Korja R, Ekholm E, Karlsson H. Cohort Profile: The FinnBrain Birth Cohort Study (FinnBrain). Int J Epidemiol. 2018; 47(1): 15-16j. https://doi.org/10.1093/ije/dyx173

24. Knickmeyer RC, Gouttard S, Kang C, Evans D, Wilber K, Smith JK, Gilmore JH. A structural MRI study of human brain development from birth to 2 years. J Neurosci. 2008; 28(47): 12176-12182.

https://doi.org/10.1523/JNEUROSCI.3479-08.2008

25. Kocevska D, Rijlaarsdam J, Ghassabian A, Jaddoe VW, Franco OH, Verhulst FC, Tiemeier H. Early Childhood Sleep Patterns and Cognitive Development at Age 6 Years: The Generation R Study. J Pediatr Psychol. 2017; 42(3): 260268. https://doi.org/10.1093/jpepsy/jsv168

26. Kochanska G, Murray KT, Harlan ET. Effortful control in early childhood: continuity and change, antecedents, and implications for social development. Dev Psychol. 2000: 36(2): 220-232.

27. Lam JC, Mahone EM, Mason T, Scharf SM. The effects of napping on cognitive function in preschoolers. J Dev Behav Pediatr. 2011; 32(2): 90-97. https://doi.org/10.1097/DBP.0b013e318207ecc7

28. Leng Y, Cappuccio FP, Wainwright NWJ, Surtees PG, Luben R, Brayne C, Khaw K-T. Sleep duration and risk of fatal and nonfatal stroke: a prospective study and meta-analysis. Neurology. 2015; 84(11); 1072-1079. https://doi.org/10.1212/WNL.0000000000001371

29. Makela TE, Peltola MJ, Saarenpaa-Heikkila O, Himanen S-L, Paunio T, Paavonen EJ, Kylliainen A. Night Awakening and Its Association With Executive Functioning Across the First Two Years of Life. Child Dev. 2020; 91(4): e937e951. https://doi.org/10.1111/cdev.13326

30. Medic G, Wille M, Hemels ME. Short- and long-term health consequences of sleep disruption. Nat Sci Sleep. 2017; 9: 151-161. https://doi.org/10.2147/NSS.S134864

31. Minde K, Popiel K, Leos N, Falkner S, Parker K, Handley-Derry M. The evaluation and treatment of sleep disturbances in young children. J Child Psychol Psychiatry 1993; 34(4): 521-533. https://doi.org/10.1111/j.14697610.1993.tb01033.x

32. Mindell JA, Leichman ES, DuMond C, Sadeh A. Sleep and Social-Emotional Development in Infants and Toddlers. J Clin Child Adolesc Psychol. 2017; 46(2): 236-246. https://doi.org/10.1080/15374416.2016.1188701

33. Mindell JA, Owens J, Alves R, Bruni O, Goh DYT, Hiscock H, Sadeh A. Give children and adolescents the gift of a good night's sleep: a call to action. Sleep Med. 2011; 12(3): 203-204. https://doi.org/10.1016/j.sleep.2011.01.003 
34. Molfese VJ, Rudasill KM, Prokasky A, Champagne C, Holmes M, Molfese DL, Bates JE. Relations Between Toddler Sleep Characteristics, Sleep Problems, and Temperament. Dev Neuropsychol. 2015; 40(3): 138-154. https://doi.org/10.1080/87565641.2015.1028627

35. Murray E, Fernandes M, Newton CRJ, Abubakar A, Kennedy SH, Villar J, Stein A. Evaluation of the INTERGROWTH21st Neurodevelopment Assessment (INTER-NDA) in 2 year-old children. PloS One. 2018; 13(2): e0193406. https://doi.org/10.1371/journal.pone.0193406

36. Paavonen EJ, Morales-Munoz I, Polkki P, Paunio T, Porkka-Heiskanen T, Kylliainen A, Saarenpaa-Heikkila 0. Development of sleep-wake rhythms during the first year of age. J Sleep Res. 2019: e12918. https://doi.org/10.1111/jsr.12918

37. Paavonen J, Saarenpää-Heikkilä O, MoralesMunoz I, Virta M, Häkälä N, Pölkki P, Karlsson L. Normal sleep development in infants: findings from two large birth cohorts. Sleep Med. 2020; 69: 145-154. https://doi.org/https://doi.org/10.1016/j.sleep.2020.01.009

38. Sadeh A. A brief screening questionnaire for infant sleep problems: validation and findings for an Internet sample. Pediatrics. 2004; 113(6): e570-7.

39. Sadeh A, De Marcas G, Guri Y, Berger A, Tikotzky L, Bar-Haim Y. Infant Sleep Predicts Attention Regulation and Behavior Problems at 3-4 Years of Age. Dev Neuropsychol. 2015; 40(3): 122-137. https://doi.org/10.1080/87565641.2014.973498

40. Sadeh A, Gruber R, Raviv A. Sleep, neurobehavioral functioning, and behavior problems in school-age children. Child Dev. 2002; 73(2): 405-417.

41. Schilling TM, Kolsch M, Larra MF, Zech CM, Blumenthal TD, Frings C, Schachinger H. For whom the bell (curve) tolls: cortisol rapidly affects memory retrieval by an inverted U-shaped dose-response relationship. Psychoneuroendocrinology. 2013; 38(9): 1565-1572. https://doi.org/10.1016/j.psyneuen.2013.01.001

42. Shing YL, Lindenberger U, Diamond A, Li S-C, Davidson MC. Memory maintenance and inhibitory control differentiate from early childhood to adolescence. Dev Neuropsychol. 2010; 35(6): 679-697. https://doi.org/10.1080/87565641.2010.508546

43. Spinrad TL, Eisenberg N, Gaertner BM. Measures of Effortful Regulation for Young Children. Infant Mental Health J. 2007; 28(6): 606-626. https://doi.org/10.1002/imhj.20156

44. Steenari M-R, Vuontela V, Paavonen EJ, Carlson S, Fjallberg M, Aronen E. Working memory and sleep in 6- to 13year-old schoolchildren. J Am Acad Child Adolesc Psychiatry. 2003; 42(1): 85-92.

45. Taveras EM, Rifas-Shiman SL, Bub KL, Gillman MW, Oken E. Prospective Study of Insufficient Sleep and Neurobehavioral Functioning Among School-Age Children. Acad Pediatr. 2017; 17(6): 625-632. https://doi.org/10.1016/j.acap.2017.02.001

46. Thomas M, Sing H, Belenky G, Holcomb H, Mayberg H, Dannals R, Redmond D. (2000). Neural basis of alertness and cognitive performance impairments during sleepiness. I. Effects of $24 \mathrm{~h}$ of sleep deprivation on waking human regional brain activity. J Sleep Res. 2000; 9(4): 335-352.

47. Turnbull K, Reid GJ, Morton JB. Behavioral Sleep Problems and their Potential Impact on Developing Executive Function in Children. Sleep. 2013; 36(7): 1077-1084. https://doi.org/10.5665/sleep.2814

48. Villar J, Fernandes M, Purwar M, Staines-Urias E, Di Nicola P, Cheikh Ismail L, Kennedy S. Neurodevelopmental milestones and associated behaviours are similar among healthy children across diverse geographical locations. Nat Commun. 2019; 10(1): 511. https://doi.org/10.1038/s41467-018-07983-4

49. Weissbluth M. Naps in children: 6 months-7 years. Sleep. 1995; 18(2): 82-87. https://doi.org/10.1093/sleep/18.2.82 
50. Wiebe SA, Espy KA, Charak D. Using confirmatory factor analysis to understand executive control in preschool children: I. Latent structure. Dev Psychol. 2008; 44(2): 575-587. https://doi.org/10.1037/0012-1649.44.2.575

\section{Figures}

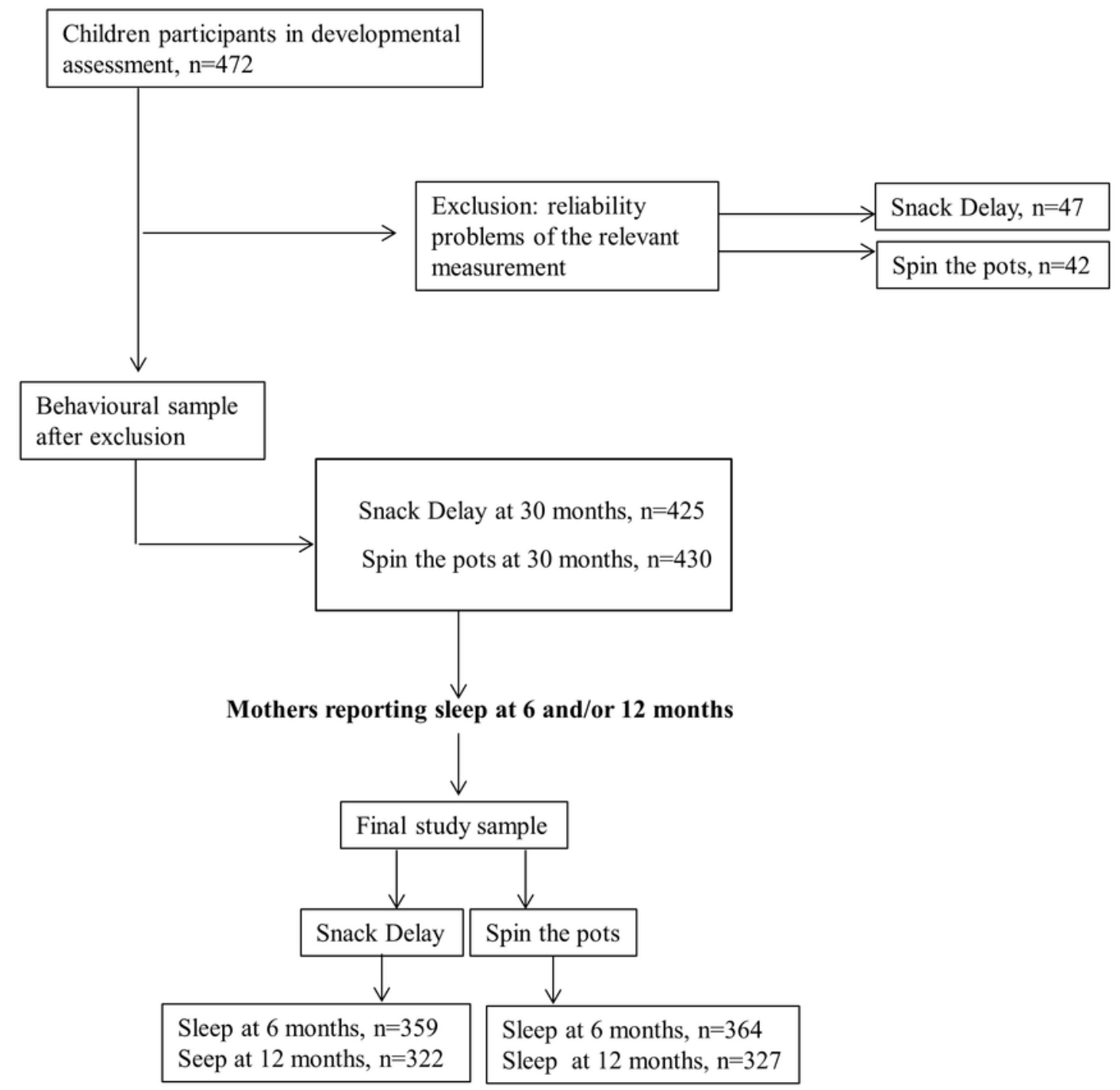

Figure 1

Flowchart of sampling procedure and exclusion criteria. This figure shows how the final sample for this study was selected. Initially, 472 children participated in the developmental assessment and from them, 47 children in Snack Delay and 42 children in Spin the Pots were excluded from the final analyses due to reliability problems of the relevant measurement. Furthermore, only those children of the remaining sample whose mothers reported on sleep at 6 and/or12 months were included in the final analyses, resulting in the following samples: i) for IC task, 359 infants with sleep questionnaire at 6 months and 322 with sleep questionnaire at 12 months; and ii) for WM task at 30 months, 364 infants with sleep questionnaire at 6 months and 327 with sleep questionnaire at 12 months. 

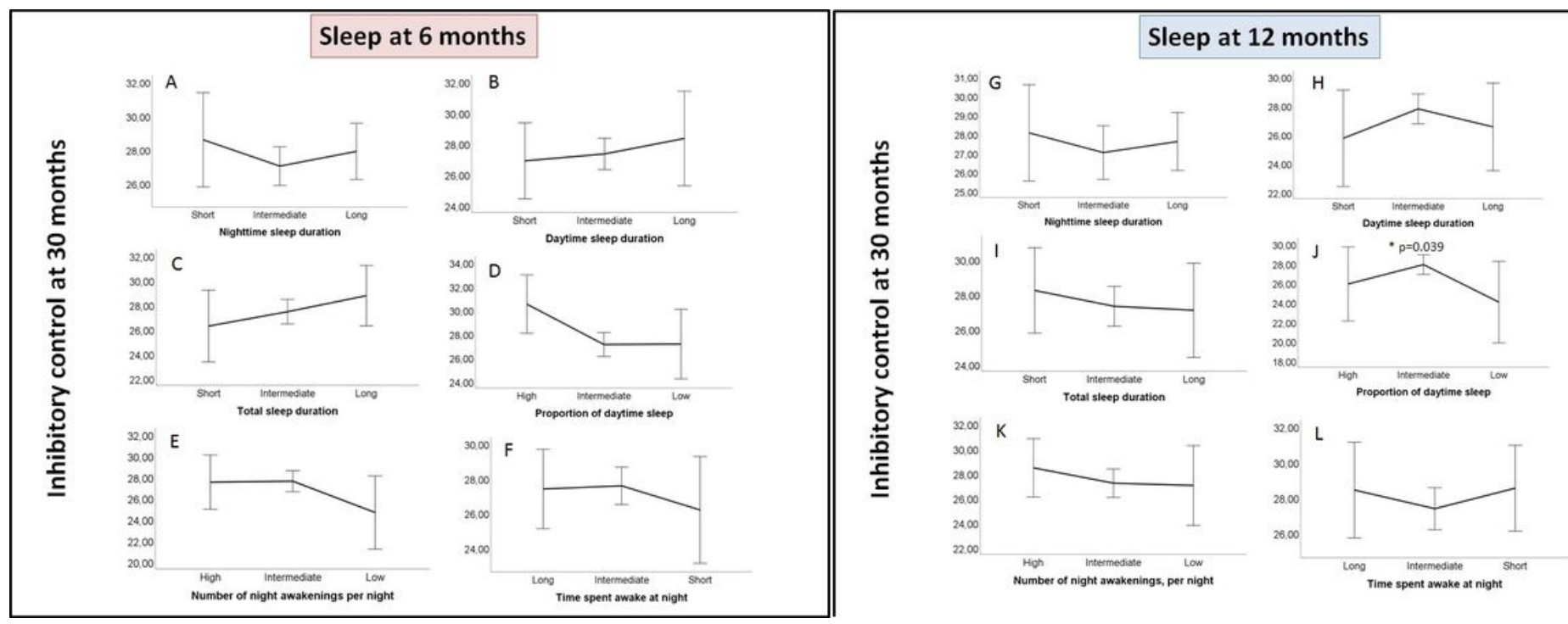

\section{Figure 2}

Estimated Marginal Means for Snack Delay, Hands version task at 30 months, for each sleep group at 6 and 12 months. This graph represents the Estimated Marginal Means for Inhibitory control at 30 months, for each sleep variable based on the 10th, 10-90th and 90th percentiles, during the first year of life (6 and 12 months). Y axis represents the estimated marginal means for the inhibitory control measure at 30 months and $\mathrm{X}$ axis the three sleep groups. Error bars represent the $95 \%$ Confidence Interval. Graphs A-F refer to sleep at 6 months, while graphs G-L refer to sleep at 12 months. The sleep variables represented here are: nighttime sleep duration (A and $G$ ), daytime sleep duration $(B$ and $H)$, total sleep duration ( $C$ and $I$ ), proportion of daytime sleep ( $D$ and $J$ ), number of night awakenings per night ( $\mathrm{E}$ and $\mathrm{K})$ and time awake during night ( $\mathrm{F}$ and $\mathrm{L})$. At 6 months, we can observe a clear linear association between total sleep duration (C) and IC, while a clear inverted U-shaped association was found between nighttime sleep duration (A) and IC. Finally, a trend towards an existing U-shaped association was found in daytime sleep duration (B) and proportion of daytime sleep (D), and towards and inverted U-shaped association in number of night awakenings (E) and time awake at night (F). Concerning the time point of 12 months, more clear associations were observed. A linear association between total sleep duration (I) and IC was found. Inverted U-shaped associations were reported in daytime sleep duration $(\mathrm{H})$ and proportion of daytime sleep $(\mathrm{J})$, while a trend towards U-shaped relation was found in nighttime sleep duration (G), number of night awakenings (K) and time awake at night (L).
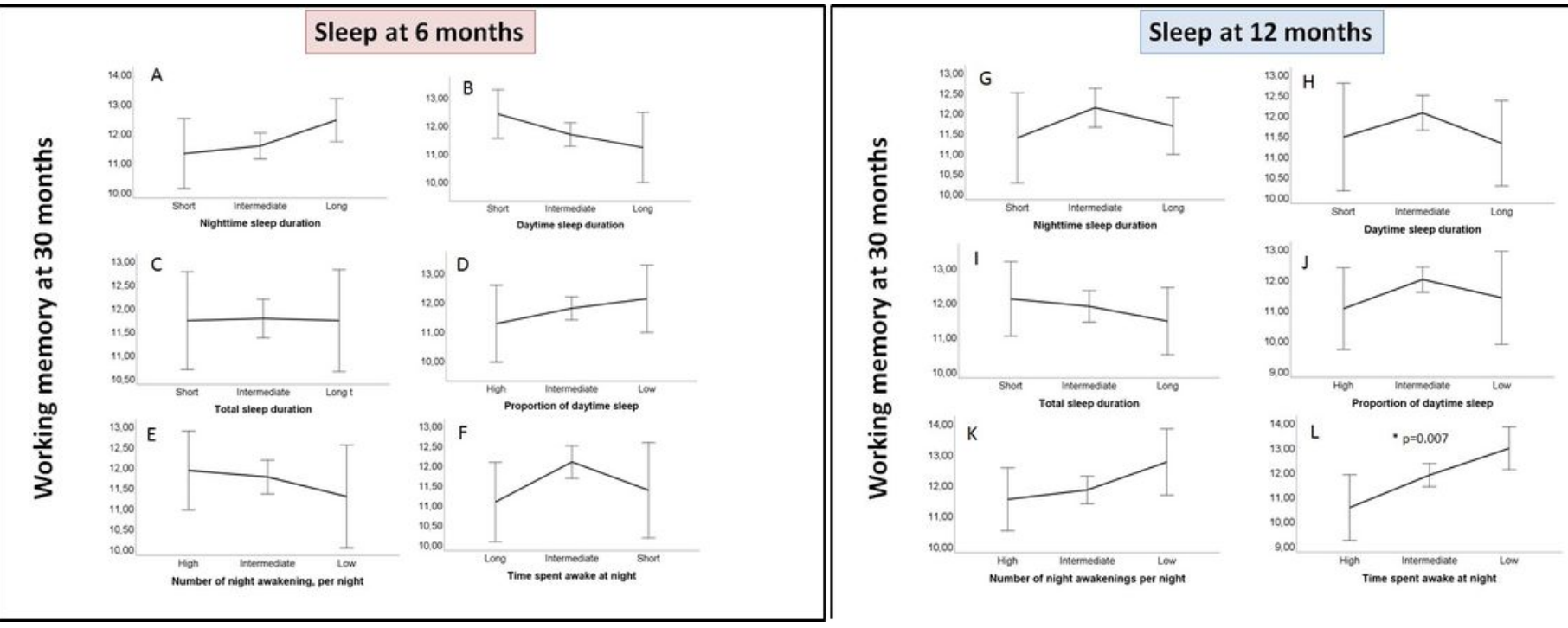

Figure 3 
Estimated Marginal Means for Spin the Pots task at 30 months, for each sleep group at 6 and 12 months. This graph represents the Estimated Marginal Means for Working Memory at 30 months, for each sleep variable based on the 10th, 10-90th and 90th percentiles, during the first year of life (six and 12 months). Y axis represents the estimated marginal means for the working memory measure at 30 months and $X$ axis the three sleep groups. Error bars represent the $95 \%$ Confidence Interval. Graphs A-F refer to sleep at 6 months, while graphs G-L refer to sleep at 12 months. The sleep variables represented here are: nighttime sleep duration ( $A$ and $G)$, daytime sleep duration ( $B$ and $H)$, total sleep duration ( $C$ and $\mathrm{I}$ ), proportion of daytime sleep ( $D$ and $\mathrm{J}$ ), number of night awakenings per night ( $E$ and $\mathrm{K}$ ) and time awake during night ( $F$ and $L)$. At 6 months we can observe that almost all the relations between sleep and WM are linear (A-E), while an inverted U-shaped association between time spent awake at night $(\mathrm{F})$ and WM was reported. At 12 months, linear associations were only found in total sleep duration (I) and time awake at night $(\mathrm{L})$, with a tendency towards linearity in number of night awakenings $(K)$. The associations between nighttime sleep duration (G), daytime sleep duration $(\mathrm{H})$ and proportion of daytime sleep duration $(\mathrm{J})$ with WM at 30 months follow an inverted U-shaped association. 